\title{
Toward Understanding the Thermodynamics of TALSPEAK Process -- Medium Effects on Actinide Complexation
}

\section{Actinides 2009}

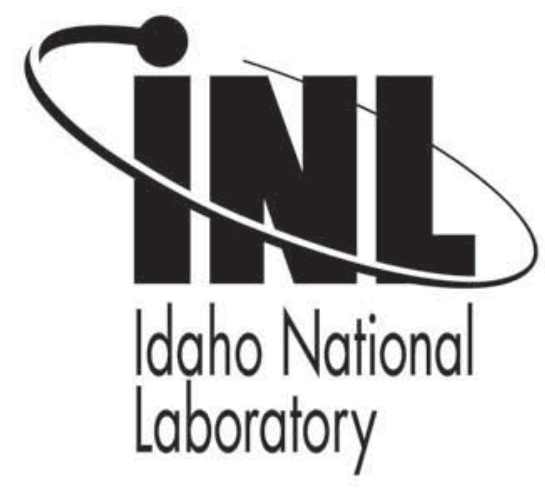

P. R. Zalupski

K. L. Nash

Y. Nakamura

M. Yamamoto

L. R. Martin

July 2009

This is a preprint of a paper intended for publication in a journal or proceedings. Since changes may be made before publication, this preprint should not be cited or reproduced without permission of the author. This document was prepared as an account of work sponsored by an agency of the United States Government. Neither the United States Government nor any agency thereof, or any of their employees, makes any warranty, expressed or implied, or assumes any legal liability or responsibility for any third party's use, or the results of such use, of any information, apparatus, product or process disclosed in this report, or represents that its use by such third party would not infringe privately owned rights. The views expressed in this paper are not necessarily those of the United States Government or the sponsoring agency. 


\title{
Toward understanding the thermodynamics of TALSPEAK process. Medium effects on actinide complexation
}

\author{
P R Zalupski, ${ }^{1,2,4}$ K L Nash, ${ }^{2}$ Y Nakamura, ${ }^{3}$ M Yamamoto ${ }^{3}$ and L R Martin ${ }^{1}$ \\ ${ }^{1}$ Idaho National Laboratory, Aqueous Separations and Radiochemistry Department, \\ Idaho Falls, ID, 83415, USA \\ ${ }^{2}$ Washington State University, Chemistry Department, Pullman, WA, 99164, USA \\ ${ }^{3}$ Japan Atomic Energy Agency, Tokai Reprocessing Technology Development Center, \\ Tokai-mura, Naka-Gun, Ibaraki-pref, 319-1194 Japan \\ E-mail: peter.zalupski@inl.gov
}

\begin{abstract}
The ingenious combination of lactate and diethylenetriamine-N,N,N',N",N"pentaacetic acid (DTPA) as an aqueous actinide-complexing medium forms the basis of the successful separation of americium and curium from lanthanides known as the TALSPEAK process. While numerous reports in the prior literature have focused on the optimization of this solvent extraction system, considerably less attention has been devoted to the understanding of the basic thermodynamic features of the complex fluids responsible for the separation. The available thermochemical information for both lactate and DTPA protonation and metal complexation reactions are representative of the behavior of these ions under idealized conditions. Significant departures from the speciation predicted based on reported thermodynamic values may be present in the TALSPEAK aqueous environment. Thus, thermodynamic parameters describing the separation chemistry of this process require further examination at conditions significantly removed from conventional ideal systems commonly employed in fundamental solution chemistry. In this report, calorimetric studies of lactate protonation equilibrium illustrate a distinct influence of nitrate, perchlorate, methylsulfonate and triflate on the thermodynamic parameters associated with TALSPEAK aqueous chemistry. Triflate is identified a closer non-complexing representative of lactate solution chemistry. The reported initial two stability constants for the complexation of americium and neodymium by lactate in triflate medium suggest that the identified medium effects do not significantly affect the speciation.
\end{abstract}

\section{Introduction}

A recent burst of research activity related to the TALSPEAK process (Trivalent Actinide - Lanthanide Separation by Phosphorous reagent Extraction from Aqueous Komplexes) stems from its consideration as part of the advanced aqueous reprocessing campaign to close the nuclear fuel cycle, i.e. the UREX+ process. TALSPEAK successfully separates trivalent lanthanides from trivalent actinides by taking advantage of actinide complex formation in the aqueous phase. An aminopolycarboxylate complexing agent, diethylenetriamine-N,N,N',N",N"-pentaacetic acid, DTPA, present in a lactate buffer solution functions as a holdback reagent for actinides, while lanthanides are extracted into the organic phase by a powerful cation exchanger, di(2-ethylhexyl)phosphoric acid,

4 To whom any correspondence should be addressed. 
HDEHP. Although still not fully proven, the accomplished separation has been attributed to the enhanced spatial extension of $5 f$ element electron shell, allowing stronger interactions with complexants containing "soft" donor groups. [1] The initial report of TALSPEAK carried out an efficient inter-group separation (i.e. $\mathrm{Ln} / \mathrm{An}$ ) with the lowest separation factor of around 10 between neodymium and californium. [2] The chemistry of TALSPEAK has been thoroughly investigated since, both, on the laboratory bench and in the pilot scale demonstrations. Nilsson and Nash have recently reviewed the history of TALSPEAK characterization efforts, [3] and, while the majority of the prior literature has developed a substantial library of information, the main focus was the optimization of the extraction process. Recent inclusion of TALSPEAK into the UREX+ framework prompted new investigative efforts focused on process control, predictive modelling and thermodynamic understanding of the chemistry that drives the process. [4] This approach builds on the recognized effectiveness of the separation task, but targets a more application-based set of criteria like the robustness of the process, response to off-normal operating conditions and corrective actions.

The complex nature of this multi-component phase transfer system was illustrated through the attempted theoretical calculations performed by Nilsson and Nash. [4] The exercise attempted to reproduce the experimentally obtained $\mathrm{pH}$ dependency for the partitioning of several lanthanides and americium between the aqueous and organic phase of representative TALSPEAK conditions. The developed model produced a set of metal distribution values, which matched the experimental data very well at the initial stages of $\mathrm{pH}$ dependency. However, the experimental results and the calculated prediction follow opposite trends, with more significant deviation as the $\mathrm{pH}$ of the aqueous phase is increased. The authors offer several possible explanations for the divergence, including: the presence of phase-transfer and aqueous complexation equilibria unaccounted for in the model, the existence of ternary complexes, where lactate participates in metal complexation by HDEHP and/or DTPA, and questions over the validity of the thermodynamic data used to model the experiment, considering changes of the ionic strength throughout the investigated $\mathrm{pH}$ range $(2.5-4.7)$. Furthermore, the shifting balance in the $\mathrm{H}^{+} / \mathrm{Na}^{+}$lactate equilibrium is expected to influence the activity of water; however, the medium effects of bulky lactate anions are relatively unknown. The available thermochemical information of both lactate and DTPA protonation and metal complexation reactions are representative of the behaviour of these species under idealized conditions, where the ionic strength is controlled by inorganic anions such as nitrate or perchlorate. When comparing the size, symmetry and charge distribution of organic conjugate bases such as lactate and inorganic anions, significant departures from the speciation predicted based on reported thermodynamic values may be present in actual TALSPEAK aqueous environment. Our intent here is to study aqueous medium effects in TALSPEAK chemistry to deliver a more palpable measure of thermodynamic departure from the TALSPEAK-defined conditions, when lactate is substituted by a strong background electrolyte. The enthalpy of lactate protonation was studied in perchlorate, nitrate, methylsulfonate and triflate media using microcalorimetry. The identified $\Delta \mathrm{H}$ value, when compared to that obtained when studying lactate protonation in $1.0 \mathrm{M}$ sodium lactate media, yielded a thermodynamic difference, $\Delta(\Delta \mathrm{H})$, indicating the influence of medium on the studied equilibrium. New stability constants for lactate protonation and lactate complexation of americium and neodymium were studied in the solution of the electrolyte, which, based on the calorimetric studies, was identified as a better representative of lactate activity effects in solution.

\section{Experimental}

Neodymium oxide, $\mathrm{Nd}_{2} \mathrm{O}_{3}$, was $99.999 \%$ pure from Rare Earth Products, Inc. Neodymium triflate stock solution was prepared by reacting the metal oxide with 5.9 equivalents of triflic acid, obtained from Sigma-Aldrich as $99+\%$ pure material. The application of heat promoted a full dissolution of oxide in deionized water slurry. Excess lanthanide oxide was filtered through a $0.2 \mu \mathrm{m}$ syringe filter and the filtrate was diluted with $1 \mathrm{mM}$ triflic acid solution. Metal concentration in the stock solution was determined by cation exchange using a Dowex 50X8 resin (Sigma Aldrich), after the initial concentration of acid was obtained. Americium-243 in $6 \mathrm{M}$ nitric acid was obtained from laboratory 
stocks. Approximately 6 grams of $15.7 \mathrm{mM}$ stock solution of americium was passed through Eichrom's two DGA resin cartridges to exchange the $6 \mathrm{M} \mathrm{HNO}_{3}$ matrix for dilute hydrochloric acid. The eluted solution was evaporated to near-dryness. Several evaporation cycles of added deionized water followed to reduce the acid content. Aqueous metal ion solutions were prepared using water from a LABCONCO water purification system. The sodium lactate solution (mixture of $d / l$ isomers at $50 \% \mathrm{w} / \mathrm{v}$ ) was obtained from Acros Organics and standardized using cation exchange resin. Sodium salts of perchloric, nitric and trifluoromethanesulfonic acid were obtained from Ricca Chemical Company (recrystallized), Fluka (99.999\%), TCI America (99.86\%), respectively. Concentrated sodium salt solutions were prepared, checked for acidic impurities and standardized using cation exchange resin. In methanesulfonate case the acid obtained from Sigma-Aldrich $(99.8 \%$ ) was neutralized with $50 \% \mathrm{w} / \mathrm{w} \mathrm{NaOH}$ (Sigma-Aldrich) to prepare an aqueous sodium salt solution. The sodium salt of trifluoromethanesulfonic acid was recrystallized twice from water and the stock solution was standardized using cation exchange resin. The concentrated perchloric, nitric and triflic acids were purchased from GFS Chemicals $(70.6 \% \mathrm{w} / \mathrm{v})$, J.T. Baker $(69.8 \% \mathrm{w} / \mathrm{v})$, and Sigma-Aldrich $(99 \%)$, respectively. All base solutions were prepared from $50 \% \mathrm{w} / \mathrm{w} \mathrm{NaOH}$ and standardized against the primary standard, potassium hydrogen phthalate, which was stored in an oven at $90^{\circ} \mathrm{C}$.

Microcalorimetric measurements were performed using isothermal titration microcalorimeter (CSC Model 4200, TA Instruments, New Castle, DE) to determine the enthalpies of lactate protonation. The operation and calibration procedures for the calorimeter were described previously. [5] Calorimetric titrations of sodium lactate to measure heats of protonation were conducted on $1 \mathrm{~mL}$ of solutions. Typical protonation experiment followed a series of additions of $0.1-0.2 \mathrm{M}$ acid into 10-20 mM sodium lactate solution, and compared to the result of the equivalent titration of $1.0 \mathrm{M}$ sodium lactate. The ionic strength was controlled at $1.0 \mathrm{M}$ with the background electrolyte under investigation. Multiple blank experiments were carried out. Titrant dilution experiments were carried out with its additions into $1.0 \mathrm{M}$ sodium salt solution. Anion dilution was also explored by titrating $1.0 \mathrm{M}$ sodium salt into $1.0 \mathrm{M}$ sodium lactate. Figure 1 shows the schematic representation of the performed calorimetric experiments to summarize the lactate protonation study. The measurement of $\mathrm{pH}$ throughout the course of each calorimetric titration was performed by repeating the titration in a small vessel outside the calorimeter. All conditions of the calorimetric experiments were replicated and the titrant was delivered using calorimeter's injection port. The $\mathrm{pH}$ of the sodium lactate solution located in the water-jacketed beaker at $25{ }^{\circ} \mathrm{C}$ was measured after each injection. The $\mathrm{pH}$ measurement was followed with a Ross semimicro glass combination electrode filled with $3 \mathrm{M} \mathrm{NaCl}$ (ThermoOrion). The electrode was calibrated to an operational $\mathrm{p}[\mathrm{H}]$ scale $\left(\mathrm{p}[\mathrm{H}]=-\log \left[\mathrm{H}^{+}\right]\right.$, where $\left[\mathrm{H}^{+}\right]$is the molar hydrogen ion concentration in solution) by titration of $\mathrm{H}^{+}\left(\mathrm{salt}^{-}\right)$with $\mathrm{NaOH}$ at $1.0 \mathrm{M}$ ionic strength and $25.0 \pm 0.1{ }^{\circ} \mathrm{C}$. Under these conditions, the measured $\mathrm{p}[\mathrm{H}]$ was accurate to $\pm 0.005 \mathrm{p}[\mathrm{H}]$ units. The conductivity of all sodium lactate solutions was measured using a Mettler Toledo MultiSeven meter. The formation enthalpies of the $\mathrm{Nd}^{3+}$ and $\mathrm{Am}^{3+}$ complexes were determined by duplicate titrations of 1 $\mathrm{mL}$ solutions containing $5 \mathrm{mM}$ metal with $0.1 \mathrm{M}$ lactate solution.

Potentiometric titrations were conducted with a Mettler Toledo T50 autotitrator equipped with acid and base $5 \mathrm{~mL}$ burettes. The microtitration vessel and a Mettler Toledo combined micro glass $\mathrm{pH}$ electrode with a ceramic frit were used to reduce sample volumes. The calibration of the micro electrode matched that performed for the Ross combination electrode.

\section{Results}

Our initial thermodynamic studies attempted to generate a "scale" for medium effects expected when either speciation or thermochemistry of TALSPEAK system is studied using a conventional ideal systems commonly employed in fundamental solution chemistry. A traditional approach in the determination of stability constants employs a strong background electrolyte to control the ionic strength. For example, one would expect changes in the solution chemistry when studying a $1.0 \mathrm{M}$ lactate buffer versus a dilute lactate solution controlled by a swamping concentration of sodium perchlorate. The measurement of $\mathrm{pH}$ based on operational $\mathrm{p}[\mathrm{H}]$ scale in the perchlorate medium may 
be skewed by the changing electrode junction potential arising upon transfer into the $1.0 \mathrm{M}$ lactate medium. The species distribution in TALSPEAK system, i.e. metal-lactate and metal-DTPA complexes, as well as the protonation equilibria, may be rearranged based on the water-structuring properties of a particular electrolyte. Our thermochemical comparison of the lactate protonation equilibrium, studied directly, in concentrated solution $(1.0 \mathrm{M})$, and conventionally, in diluted $(0.02 \mathrm{M}$, $I=1.0 \mathrm{M}$ ) solution, yielded a trend of thermodynamic deviations caused by changes in the aqueous composition. The trend agreed well with the fundamental physico-chemical properties of aqueous solutions such as ion mobility and conductivity, suggesting a direct medium effect.

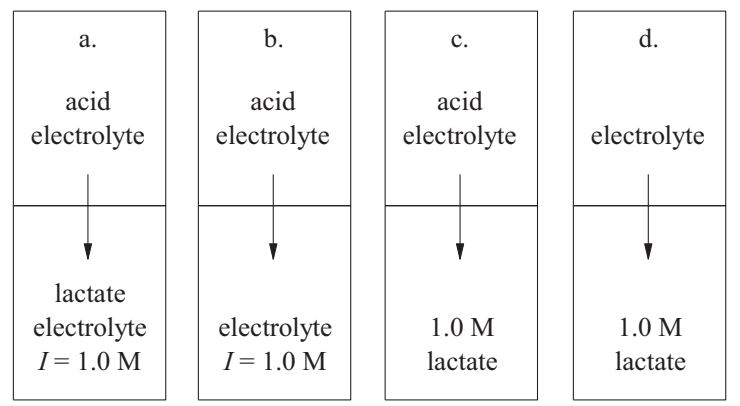

Figure 1. Experimental sequence of calorimetric runs designed to explore medium effects on lactate protonation equilibrium, a) lactate protonation in background electrolyte solution, b) titrant dilution, c) lactate protonation at TALSPEAK-like conditions, d) anion dilution.

\subsection{Medium effects on lactate protonation}

Typical design of the calorimetric investigation of lactate protonation in a chosen background electrolyte system is summarized in Figure 1. Four different calorimetric titration experiments, performed at least in duplicate, composed the study for each electrolyte system under investigation.
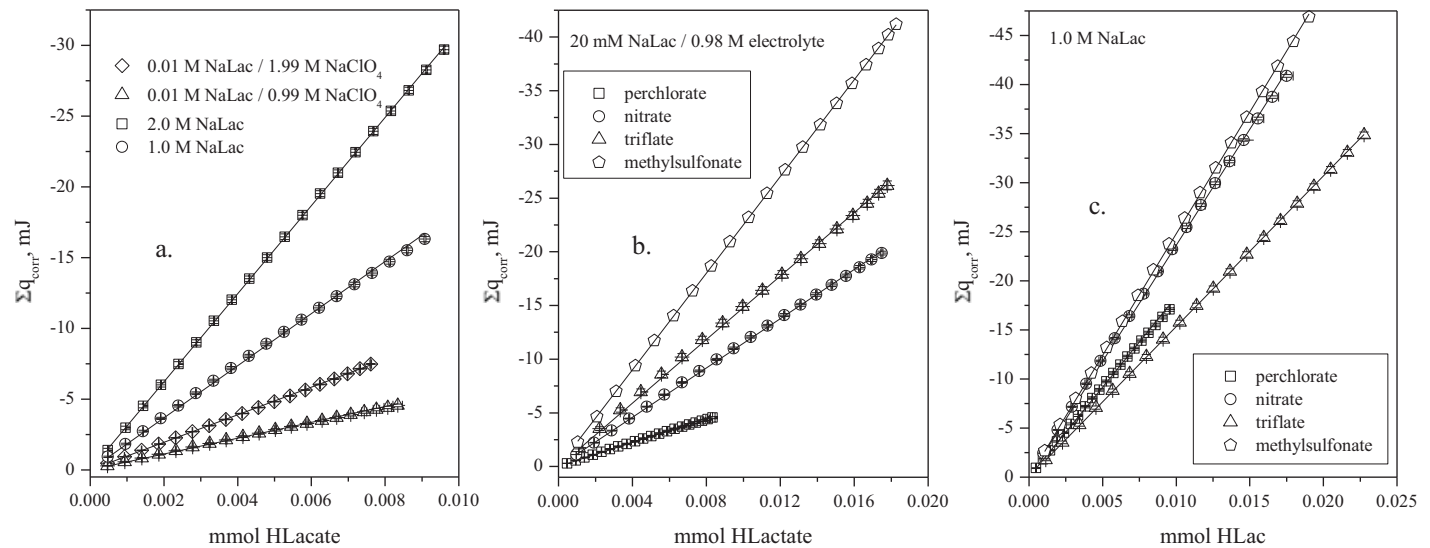

Figure 2. Calorimetric investigations of the medium effects of perchlorate, nitrate, triflate and methylsulfonate on the enthalpy of lactate protonation. (a) Effect of ionic strength. (b) Calorimetric titrations using background electrolyte. (c) Calorimetric titrations in $1.0 \mathrm{M}$ lactate.

Figure 2a shows the cumulative heat of lactate protonation, corrected for the dilution events, studied in the perchlorate medium of two different concentrations. The lactate protonation enthalpy was calculated by linear regression according to $\Delta \mathrm{H}=\Sigma \mathrm{q}_{\mathrm{corr}} / \mathrm{m}$, where $\mathrm{m}$ represents the number of moles of protonated lactate. The observed thermodynamic deviation from TALSPEAK-like conditions caused by the electrolyte was estimated by the difference in the enthalpies of lactate protonation, $\Delta(\Delta \mathrm{H})$, studied in electrolyte medium or pure lactate. The effect of the ionic strength on the protonation 
equilibria is clearly visible and will be discussed in the following section. Figures $2 \mathrm{~b}$ and $2 \mathrm{c}$ summarize all thermochemical studies of lactate protonation for the $1.0 \mathrm{M}$ ionic strength in nitrate, perchlorate, triflate and methylsulfonate media. The comparison of the calorimetric results for the lactate protonation in background electrolyte solutions, $\Delta \mathrm{H}_{1}$, (Figure $2 \mathrm{~b}$ ) with those performed at TALSPEAK-like conditions, $\Delta \mathrm{H}_{2}$, (Figure 2c) identifies thermodynamic differences caused by using all strong background electrolytes. Table 1 lists all enthalpy values for the studied lactate solutions.

Table 1. Enthalpies of lactate protonation investigated using background electrolyte, $\Delta \mathrm{H}_{1}$, and directly in lactate solutions, $\Delta \mathrm{H}_{2}$, reported at $25^{\circ} \mathrm{C}$, with uncertainties at $\pm 2 \sigma$.

\begin{tabular}{lcccc}
\hline Electrolyte & $I, \mathrm{M}$ & $\Delta \mathrm{H}_{1}(\mathrm{~kJ} /$ mole $)$ & $\Delta \mathrm{H}_{2}(\mathrm{~kJ} /$ mole $)$ & $\Delta(\Delta \mathrm{H}), \mathrm{kJ} / \mathrm{mole}$ \\
\hline perchlorate & 2.0 & $-0.98 \pm 0.02$ & $-3.11 \pm 0.04$ & 2.13 \\
perchlorate & 1.0 & $-0.56 \pm 0.02$ & $-1.84 \pm 0.03$ & 1.28 \\
nitrate & 1.0 & $-1.15 \pm 0.02$ & $-2.36 \pm 0.03$ & 1.21 \\
triflate & 1.0 & $-1.48 \pm 0.04$ & $-1.53 \pm 0.03$ & 0.05 \\
methylsulfonate & 1.0 & $-2.25 \pm 0.05$ & $-2.48 \pm 0.03$ & 0.23 \\
\hline
\end{tabular}

\subsection{Thermodynamics of americium(III) and neodymium(III) complexation by lactate}

Our exploration of the medium effects on lactate protonation equilibrium identified triflate, Tf, as a possible analog of lactate in solution. The lactate protonation thermochemistry showed no significant variation whether studied in $1.0 \mathrm{M}$ lactate or when using sodium triflate as a background electrolyte. Taking advantage of the non-coordinating properties of a triflate anion the thermodynamics of americium(III) and neodymium(III) complexation by lactate were studied in this medium. The intent of this study was to determine how the speciation of the TALSPEAK-related equilibria are affected when examined in solutions far removed from the original conditions of the separation process. A plot of typical data obtained from potentiometric titrations of $\mathrm{Nd}^{3+}$ and $\mathrm{Am}^{3+}$ with lactate is shown in Figure 3. The titrations of metal lactate complexes with acid are compared with the titration curve when no metal is present in solution. The potentiometric data was fit using the program Psequad [6] to determine the stability constants for the protonation and complexation reactions. Our initial determination of the autoprotolysis constant yielded a $\mathrm{pK}_{\mathrm{w}}$ value of 13.73. A variety of complex species were tested, including the hydrolyzed and polynuclear species. The best fit was afforded when successive mononuclear species are used in the model for both $\mathrm{Am}$ and $\mathrm{Nd}$. For the titrations containing neodymium, three metal-to-ligand ratios were analyzed to confirm the constants. Although the iterative routine minimized the error square sum parameter using the system containing three complex species, the value of the third constant, $\beta_{103}$, was elevated for all data sets. Consequently, the results of the analysis imply a stronger nature of the third lactate complexation reaction, $\mathrm{K}_{1}$, relative to $\mathrm{K}_{2}$. It is our conclusion that the third constant is influenced by the existence of the fourth complexation reaction. All attempts to resolve the $\beta_{103}$ and $\beta_{104}$ constants using the Psequad fitting routine were unsuccessful due to the very dilute conditions of our potentiometric experiments. To confirm this hypothesis we are currently performing a series of spectrophotometric titrations in the upcoming future for both the Am and Nd systems. Hence, at this time we report the thermodynamic data for the initial two complexation reactions. The results of the potentiometric titrations are summarized in Table 2. 


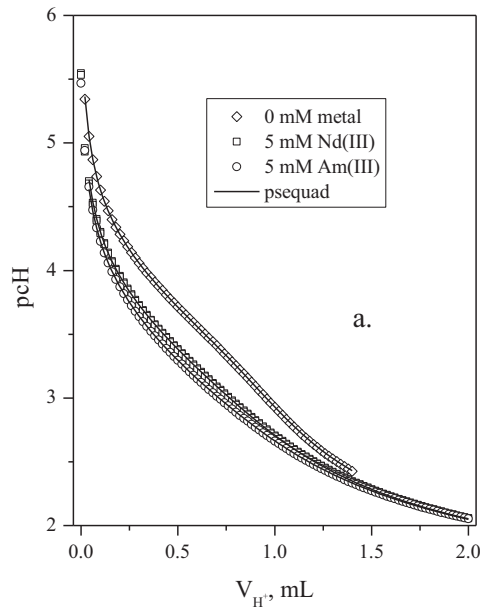

Figure 3. (a) Potentiometric titrations of lactate complexes with neodymium and americium complexes, $T=25^{\circ} \mathrm{C}, I=1.0$ $\mathrm{M}$ sodium triflate, $V_{\text {init }}=9.994 \mathrm{~mL}$, titrant: $0.1006 \mathrm{M} \mathrm{HTf}$, titrand: $5.00 \mathrm{mM} \mathrm{Nd}^{3+} / 10.3 \mathrm{mM}$ lactate, or $5.10 \mathrm{mM} \mathrm{Am}^{3+}$ / $10.04 \mathrm{mM}$ lactate.

Table 2. Stability constants for lactate protonation and complexation with $\mathrm{Am}(\mathrm{III})$ and $\mathrm{Nd}(\mathrm{III})$, reported at $25^{\circ} \mathrm{C}, \quad I=1.0$ M NaTf, with uncertainties at $\pm 2 \sigma$.

\begin{tabular}{cccc}
\hline & $j$ & $\log \beta_{10 j}$ & $\begin{array}{c}\Delta \mathrm{G}_{j} \\
\mathrm{~kJ} / \mathrm{mol}\end{array}$ \\
\hline $\mathrm{H}^{+}+\mathrm{L}^{-}=\mathrm{HL}$ & & $3.65 \pm 0.02$ & -20.8 \\
\hline $\mathrm{Nd}^{3+}+j \mathrm{~L}^{-}=\mathrm{NdL}_{j}^{(3-j)+}$ & 1 & $2.63 \pm 0.06$ & -15.0 \\
& 2 & $4.44 \pm 0.05$ & -25.3 \\
\hline $\mathrm{Am}^{3+}+j \mathrm{~L}^{-}=\mathrm{AmL}_{j}^{(3-j)+}$ & 1 & $2.68 \pm 0.04$ & -15.3 \\
& 2 & $4.93 \pm 0.06$ & -28.1 \\
\hline
\end{tabular}

\section{Discussion}

The investigation of lactate protonation in the variety of electrolyte systems and a lactate buffer allowed the identification of a fundamental relationship. The observed thermochemical deviation, described as a difference between the enthalpy of lactate protonation studied at the idealized and buffered conditions, decreases together with the ionic conductivity of the electrolyte solution, $\Lambda$. Figure 4 illustrates the observed correlation. Although the solutions pertaining to this study are far removed from the state of infinite dilution the conductivity measurements clearly indicate the thermochemical response of the studied equilibrium to fundamental properties of the electrolyte solution. This trend is preserved when the conductivity values are replaced with the ionic mobilities of the anions considered in this study, reported at infinite dilution. [7] The lower conductance of the lactate solution is indicative of a less hydrated, slower-moving bulky carboxylate anion. [8] While all the investigated electrolytes disrupt water structure, the relative hydrophobicity of lactate must be most influencing out of this group. The observed correlation indicates trifluoromethanesulfonate, i.e. triflate anion as a much closer representative of the physico-chemical characteristics of the lactate in solution. To visualize an explanation of this similarity we have to consider the tremendous electroninducing influence of the $-\mathrm{CF}_{3}$ substituent on the rest of the molecule. Trifluoromethyl group localizes the charge density of triflate anion affecting the overall surface charge and, as a result, its hydration and mobility. [8] The perchlorate and nitrate anions, where the central atoms are symmetrically 
surrounded by the oxygens, behave like true inorganic electrolytes, explaining the largest thermochemical deviation.

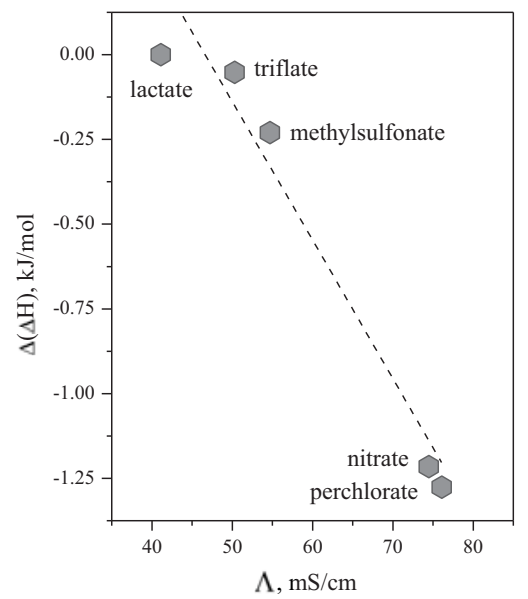

Figure 4. Thermodynamic relationship observed for the lactate protonation equilibrium when studied in a variety of electrolyte system.

The enthalpy of protonation of lactate studied in all electrolyte systems was small and exothermic, which is consistent with other members of $\alpha$-hydroxycarboxylate family. [9] The strength of lactic acid in triflate solution did not vary significantly from the reported protonation constants. [9] This observation implies that the thermodynamic equilibrium of lactate system is wellbalanced by the enthalpy-entropy compensation and the overall speciation is not affected by the nature of the medium. Similarly, the formation constants for the reported lactate complexes with $\mathrm{Nd}(\mathrm{III})$ and $\mathrm{Am}$ (III) do not imply any noticeable changes in the species distribution for this system. [10-12] The stabilities of americium-lactate complexes are similar to those of neodymium, consistently with the comparable charge densities for trivalent ions of similar ionic radii. The difference in the strength of the second complex is somewhat surprising and will be further investigated by UV-Vis spectrophotometry. The presence of the MLac ${ }_{4}{ }^{-}$ appears to complicate the identification of the third complex and requires further attention to complete the thermodynamic characterization of this system.

\section{Conclusions}

Our studies of lactate protonation revealed a distinct thermodynamic relationship between the thermochemistry of this process and the fundamental physico-chemical characteristics of the aqueous electrolyte solutions. Trifluoromethanesulfonate, i.e. triflate, was identified as a much closer analog for the solution behavior of lactate. Our initial thermodynamic studies in triflate medium reveal no significant changes in the overall speciation of the system, with or without metal in solution. Differences in the enthalpy-entropy balance for the studied equilibria were observed and will be investigated further. The thermodynamic characterization of the lactate-containing equilibria in triflate solution will be completed with future spectrophotometric titrations. However, the already assembled thermodynamic information suggests that the observed modelling divergence may not be explained by the departures from TALSPEAK-defined speciation caused by the characterization under idealized conditions.

\section{References}

[1] Jensen M 2008 Origins of $f$-element selectivity in solvent extraction Proc. International Solvent Extraction Conference ISEC 2008 (Tuscon, AZ, 15-19 September 2008) (Canadian Institute of Mining, Metallurgy and Petroleum, vol 2) ed B A Moyer (Quebec: Met Soc) pp 1081-1086.

[2] Weaver B and Kappelmann F A 1964 ORNL-3559

[3] Nilsson M and Nash K L 2007 Solvent Extr. Ion Exch. 25665

[4] Nilsson M and Nash K L 2009 Solvent Extr. Ion Exch. 27354

[5] Zalupski P R and Nash K L 2008 Solvent Extr. Ion Exch. 26514

[6] Zekany L and Nagypal I 1985 PSEQUAD: A comprehensive program for the evaluation of potentiometric and/or spectrophotometric equilibrium data using analytical derivatives Computational Methods for the Determination of Formation Constants ed D J Leggett (New York: Plenum Press)

[7] Barry P H and Lynch J W 1991 J. Membrane Biol. 121101 
[8] Robinson R A and Stokes R H 2002 Electrolyte Solutions $2^{\text {nd }}$ ed. (Mineola, NY: Dover Publications, Inc.) p 122

[9] Martell A E and Smith R M 2004 NIST Critically Selected Stability Constants of Metal Complexes Database: Version 8.0

[10] Choppin G R and Chopoorian J A 1961 J. Inorg. Nucl. Chem. 2297

[11] Bukietynska K, Mondry A and Osmeda E 1981 J. Inorg. Nucl. Chem. 431321

[12] Deelstra H and Verbeek F 1964 Anal. Chim. Acta 31251

\section{Acknowledgments}

This work was supported by the U.S. Department of Energy, Office of Nuclear Energy, under DOE Idaho Operations Office Contract DE-AC07-05ID14517. 